Document downloaded from:

http://hdl.handle.net/10251/68264

This paper must be cited as:

Beltrán, A.; Felipe Román, MJ. (2013). Solvability of normal subgroups and G-class sizes. Publicationes Mathematicae Debrecen. 83(4):1-12. http://hdl.handle.net/10251/68264.

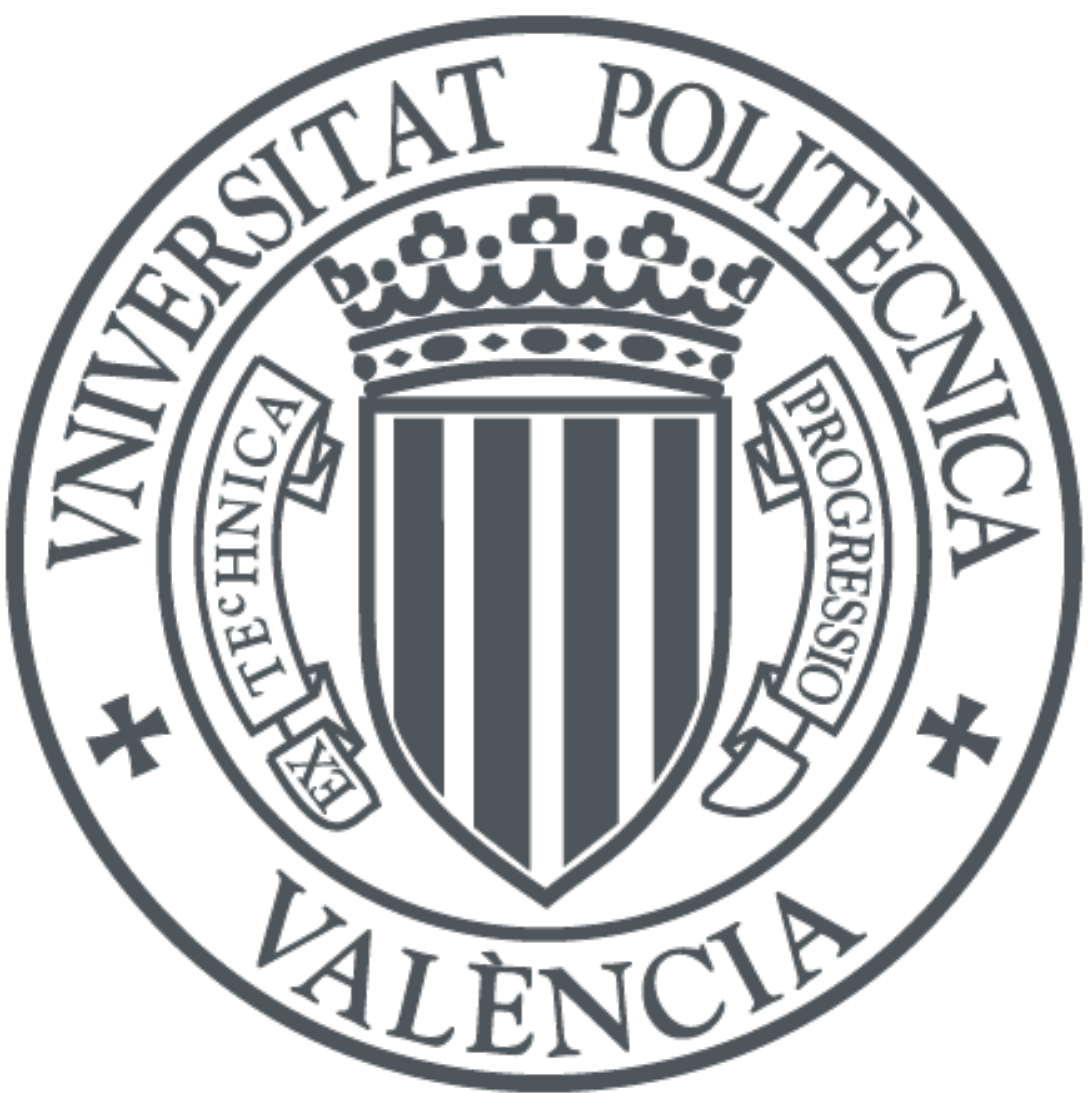

The final publication is available at

http://www.math.klte.hu/publi/contents.php?szam=83

Copyright Debreceni egyetem matematika intézet

Additional Information 


\title{
Solvability of normal subgroups and $G$-class sizes
}

\author{
By ANTONIO BELTRÁN (Castellón) and MARÍA JOSÉ FELIPE (Valencia)
}

\begin{abstract}
We study the solvability of a normal subgroup $N$ of a finite group $G$ having exactly three $G$-conjugacy class sizes. We show that if the set of $G$-class sizes of $N$ is $\left\{1, m, m p^{a}\right\}$, with $p$ a prime not dividing $m$, then $N$ is solvable. Thus, we get a partial extension for normal subgroups on N. Itô's theorem on the solvability of groups having exactly three class sizes.
\end{abstract}

\section{Introduction}

The most relevant result related to groups with three class sizes is due to N. ITô, who showed in [13] that such groups are always solvable appealing to the Feit-Thompson's theorem and some deep classification theorems by M. Suzuki. This result was simplified by J. REBMANN in [16] for $F$-groups (that is, those groups containing no pair of non-central elements $x$ and $y$ such that the centralizer of $x$ contains that of $y$ properly). Then he determined the structure of $F$-groups by using results of $\mathrm{R}$. Baer and Suzuki about groups with a non-trivial normal partition. Afterwards, A. R. CAmina proved in [7], by using the description of finite groups with dihedral Sylow 2-subgroups given by D. Gorenstein and J. H. Walter, that if $G$ does not possess the property $F$ and has three class sizes, then $G$ is a direct product of an abelian subgroup and a subgroup whose order involves

Mathematics Subject Classification: 20E45, 20 D20.

Key words and phrases: Finite groups, conjugacy class sizes, solvable groups.

This research is supported by the Valencian Government, Proyecto PROMETEO/2011/30, by the Spanish Government, Proyecto MTM2010-19938-C03-02 and the first author is also supported by grant Fundació Bancaixa P11B2010-47. The second author would like to thank the members of the Institute of Mathematics at the University of Aberdeen for their warm hospitality and for many helpful discussions during her visit to this University. 
no more than two primes. Forty years later, the structure of these groups has been completely determined (up to nilpotent groups, which in this context are $p$-groups) by S. DOLFI and E. JABARA in [8], basing their proof on the solvability of this type of groups.

Now, we focus our attention to a normal subgroup $N$ of a group $G$ and denote by $\operatorname{cs}_{G}(N)$ the set of $G$-class sizes of elements in $N$. It is natural to wonder whether the cardinality of $\operatorname{cs}_{G}(N)$, which is not related to the number of class sizes of $N$, may have a similar influence on the structure of $N$, and in fact, some recent works have given an affirmative answer. Normal subgroups having exactly two $G$-class sizes have been proved to be nilpotent [3]. Also, in [1], the structure of a normal subgroup with three $G$-class sizes, under certain arithmetical conditions on these sizes, is determined. The proof is inspired by a generalization for normal subgroups of the concept of $F$-group. The authors classify and determine the structure of $F$-normal subgroups, and as a consequence, they obtain the solvability of $F$-normal subgroups with three $G$-class sizes. In particular, they show that when $N$ is a normal subgroup of $G$ such that $\operatorname{cs}_{G}(N)=\{1, m, n\}$, where $m<n$ and $m$ does not divide $n$, then $N$ is solvable and its structure is determined.

We conjecture that the solvability of $N$ holds whenever $\left|\operatorname{cs}_{G}(N)\right|=3$, that is, the case left open in the above work also leads to solvability: If $N$ is normal in $G$ and $\operatorname{cs}_{G}(N)=\{1, m, n\}$, with $m$ dividing $n$, then $N$ is solvable. In view of Camina's work, the solution of this problem seems more difficult and it needs different techniques from those employed in [1]. In this paper, we show the following contribution to our conjecture.

Theorem A. Let $N$ be an normal subgroup of $G$, such that $\operatorname{cs}_{G}(N)=$ $\left\{1, m, m p^{a}\right\}$, with $p$ a prime and $(m, p)=1$. Then $N$ is solvable.

In order to prove Theorem A, we will make use of some key results concerning the nilpotency of normal subgroups with two $G$-class sizes, which allow us to work inductively. On the other hand, we will also employ certain result (main theorem of [2]) about the structure of normal subgroups having two $p$-regular $G$-class sizes, these are the sizes of $G$-classes whose elements have order not divisible by $p$. This will be used when $p$ is the prime appearing in the statement of Theorem A. Moreover, we develop a general result on the Schur multiplier of a simple group (Lemma 1), as well as certain properties relating the $G$-class sizes to the Fitting subgroup or the center of a normal subgroup, such as Theorems 5 and 7 or the following theorem, all of which might be useful in demonstrating the mentioned conjecture. 
Theorem B. If $N$ is a nonabelian normal subgroup of a finite group $G$ and $\left|\operatorname{cs}_{G}(N)\right|=3$, then $\mathbf{Z}(N)<\mathbf{F}(N)$.

Throughout this paper all groups are finite. If $x$ is any element of a group $G$, we denote by $x^{G}$ the conjugacy class of $x$ in $G$ and $\left|x^{G}\right|$ is called the $G$-conjugacy class size of $x$ or the index of $x$ in $G$. If $p$ is a prime number and $n$ is an integer, we use the notation $n_{p}$ for the $p$-part of $n$, and $\pi(n)$ for the set of primes dividing $n$. For the rest of notation, we will follow [12].

\section{Preliminaries}

In this section, we develop the results we are are going to use. The first lemma concerns to simple groups.

Lemma 1. A finite nonabelian simple group does not have a nontrivial conjugacy class whose size divides the order of its Schur multiplier.

Proof. Let $M(S)$ denote the Schur multiplier of a simple group $S$ (the reader is referred to Chapter 5 of [9] for a definition). We also follow this reference to compute the conjugacy class sizes of certain simples groups. First, notice that simple groups do not have conjugacy classes of prime power size distinct from 1 by Burnside's theorem [11, 15.2], so we only have to analyze those simple groups whose Schur multiplier does not have prime power order. This can only occur at most for the groups that belong to the following list (see Chapter 5 of [14]).

i) Certain linear groups: $M\left(A_{1}\left(3^{2}\right)\right)$ of order $6 ; M\left(A_{2}\left(2^{2}\right)\right)$ of order 48 ; and $M\left(A_{n}(q)\right)$ for $(n, q) \neq(1,4),(1,9),(2,2),(2,4),(3,2)$, that has order $\operatorname{gcd}(n+$ $1, q-1)$. In the first two cases the corresponding group does not have conjugacy classes of size dividing 6 and 48, respectively. In the latter case, if such a simple linear group $S$ has a conjugacy class whose size divides $\operatorname{gcd}(n+1, q-1)$, then $S$ certainly has a subgroup of index dividing $q-1$. This implies that $|S|$ must divide $(q-1)$ !. However, this cannot happen because the order of $A_{n}(q)=\operatorname{PSL}(n+1, q)$, which is

$$
\frac{\left(q^{n+1}-1\right)\left(q^{n+1}-q\right) \ldots\left(q^{n+1}-q^{n}\right)}{(q-1)(n+1, q-1)},
$$

does not divide $(q-1)$ !. In order to show it, we argue as follows. Suppose that $q=p^{f}$, for a prime $p$ and $f \geq 1$, and let $E$ denote the floor function, that is, $E(x)$ is the largest integer not greater than the real number $x$. It is an elementary property that the largest power of $p$ that divides $\left(p^{f}-1\right)$ ! is 


$$
\begin{aligned}
& E\left(\frac{p^{f}-1}{p}\right)+E\left(\frac{p^{f}-1}{p^{2}}\right)+\ldots E\left(\frac{p^{f}-1}{p^{f-1}}\right) \\
&<p^{f-1}+p^{f-2}+\ldots+p+1<p^{f}
\end{aligned}
$$

On the other hand, it is clear that $q q^{2} \ldots q^{n}=p^{\frac{n(n+1)}{2}}$ divides $\left|A_{n}(q)\right|$ and since $p^{\frac{n(n+1)}{2}} \geq p^{f}$, so the claim is proved.

ii) Some unitary groups: $M\left({ }^{2} A_{3}\left(3^{2}\right)\right)$ of order $36, M\left({ }^{2} A_{5}\left(2^{2}\right)\right)$ of order 12 , and $M\left({ }^{2} A_{n}\left(q^{2}\right)\right)$ for $(n, q) \neq(3,4),(3,9),(5,4)$, which has order $\operatorname{gcd}(n+1, q+1)$. One checks that in the first two cases there is no class size dividing 36 and 12 respectively, while in the latter case one can argue similarly as in case i) to prove the thesis of the theorem.

iii) Two orthogonal groups: $B_{2}(2)^{\prime} \cong \mathrm{A}_{6}$ and $B_{3}(3)$, both having Schur multiplier of order 6 , and with no class size dividing 6 .

iv) Three sporadic groups: $M_{22}, F i_{22}$ and $S u z$, the sporadic Suzuki group, whose Schur multipliers have order 12, 6 and 6 respectively. No class size in each of these groups divides the order of its corresponding Schur multiplier.

The following definitions and results are inspired by several theorems for ordinary classes developed by DoLFi and JABARA in [8]. We aim to extend the corresponding results for $G$-classes of normal subgroups in a group $G$.

Definition. Let $G$ be a group, $p$ a prime number and $N$ a normal subgroup of $G$. We define

and

$$
m_{p, G}(N)=\max \left\{\left|\mathbf{C}_{G}(x)\right|_{p}: x \in N \backslash \mathbf{Z}(G)\right\}
$$

$$
M_{p, G}(N)=\left\{x \in N \backslash \mathbf{Z}(G):\left|\mathbf{C}_{G}(x)\right|_{p}=m_{p, G}(N) .\right.
$$

Lemma 2. Let $N$ be a normal subgroup of a group $G$. Denote by $\bar{G}=$ $G / \mathbf{O}_{p^{\prime}}(N)$ and use the bar convention. If $g \in M_{p, G}(N)$ and $\bar{g} \notin \mathbf{Z}(\bar{G})$, then $\bar{g} \in M_{p, \bar{G}}(\bar{N})$.

Proof. We claim that for any $\bar{x} \in \bar{N} \backslash \mathbf{Z}(\bar{G})$ there exists some $y \in N \backslash \mathbf{Z}(G)$ such that $\bar{y}=\bar{x}$ and $\left|\mathbf{C}_{G}(y)\right|_{p}=\left|\mathbf{C}_{G}(x)\right|_{p}$. Let $D$ be a subgroup of $G$ such that $\bar{D}=\mathbf{C}_{\bar{G}}(\bar{x})$ and let $P$ be a Sylow $p$-subgroup of $D$. Note that $P$ acts on $N$ and in particular, acts coprimely on $\mathbf{O}_{p^{\prime}}(N)$, so by Lemma 14.1 of [11] there exists $y \in \mathbf{C}_{N}(P)$ such that $\bar{y}=\bar{x}$. Since $P \subseteq \mathbf{C}_{G}(y)$, we can take $P_{1}$ a Sylow $p$-subgroup of $\mathbf{C}_{G}(y)$ such that $P \subseteq P_{1}$. Then $\bar{P} \subseteq \overline{P_{1}} \subseteq \mathbf{C}_{\bar{G}}(\bar{y})=\bar{D}$ and since $\bar{P}$ is a Sylow $p$-subgroup of $\bar{D}$, we conclude that $\overline{P_{1}}=\bar{P}$. Furthermore, since 
$\left|P_{1}\right|=\left|\overline{P_{1}}\right|$, it follows that $P_{1}=P$ and consequently, $\left|\mathbf{C}_{G}(y)\right|_{p}=\left|\mathbf{C}_{G}(x)\right|_{p}$ as we wanted to prove. Moreover, notice that $y \notin \mathbf{Z}(G)$ because $\bar{y} \notin \mathbf{Z}(\bar{G})$.

Therefore, the above claim implies that $m_{p, \bar{G}}(\bar{N}) \leq m_{p, G}(N)$. Now, let $g \in M_{p, G}(N)$ such that $\bar{g} \notin \mathbf{Z}(\bar{G})$. Then $\left|\mathbf{C}_{\bar{G}}(\bar{g})\right|_{p} \leq m_{p, \bar{G}}(\bar{N})$. If $P$ is a Sylow $p$-subgroup of $\mathbf{C}_{G}(g)$, then $m_{p, G}(N)=|P|=|\bar{P}|$. We know that $\bar{P} \subseteq \mathbf{C}_{\bar{G}}(\bar{g})$, so $m_{p, G}(N) \leq\left|\mathbf{C}_{\bar{G}}(\bar{g})\right|_{p} \leq m_{p, \bar{G}}(\bar{N}) \leq m_{p, G}(N)$. Thus, the equality holds, whence $\bar{g} \in M_{p, \bar{G}}(\bar{N})$.

Lemma 3. Let $G$ be a finite group and $N$ a normal subgroup of $G$. Suppose that $g \in M_{p, G}(N)$ and let $P_{0}$ be a Sylow p-subgroup of $\mathbf{C}_{G}(g)$. Then $\mathbf{C}_{\mathbf{O}_{p}(N)}\left(P_{0}\right) \subseteq P_{0}$.

Proof. Let $x \in \mathbf{C}_{\mathbf{O}_{p}(N)}\left(P_{0}\right)$. If $x \in \mathbf{Z}(G)$, then it trivially lies in $P_{0}$. So, assume that $x \notin \mathbf{Z}(G)$ and consider the subgroup $\left\langle x, P_{0}\right\rangle \subseteq \mathbf{C}_{G}(x)$. Then $\left|\left\langle x, P_{0}\right\rangle\right| \leq m_{p, G}(N)=\left|P_{0}\right|$ and hence $x \in P_{0}$.

Lemma 4. Let $G$ be a finite group and $N$ a p-solvable normal subgroup of $G$. Suppose that $g$ is a $p^{\prime}$-element of $N$ such that $g \in M_{p, G}(N)$. Then $g \in \mathbf{O}_{p^{\prime}}(N)$.

Proof. We argue by induction on $|N|$. Assume first that $\mathbf{O}_{p^{\prime}}(N) \neq 1$ and consider $\bar{G}=G / \mathbf{O}_{p^{\prime}}(N)$. If $g \notin \mathbf{O}_{p^{\prime}}(N)$, then it is clear that $\bar{g} \notin \mathbf{Z}(\bar{N})$ and consequently, $\bar{g} \notin \mathbf{Z}(\bar{G})$. Then, by Lemma $2, \bar{g} \in M_{p, \bar{G}}(\bar{N})$ and by induction $\bar{g} \in \mathbf{O}_{p^{\prime}}(\bar{N})=1$, a contradiction. Thus, in this case $g \in \mathbf{O}_{p^{\prime}}(N)$.

We assume now that $\mathbf{O}_{p^{\prime}}(N)=1$. Let $P_{0}$ be a Sylow $p$-subgroup of $\mathbf{C}_{G}(g)$ and consider the action of $P_{0} \times\langle g\rangle$ on $\mathbf{O}_{p}(N)$. By Lemma 3, we know that $\mathbf{C}_{\mathbf{O}_{p}(N)}\left(P_{0}\right) \subseteq P_{0}$ and then we can apply Thompson's $P \times Q$-Lemma (see for instance [12,4.31]) to obtain $g \in \mathbf{C}_{N}\left(\mathbf{O}_{p}(N)\right)$. However, since $N$ is $p$-solvable and $\mathbf{O}_{p^{\prime}}(N)=1$, we have $\mathbf{C}_{N}\left(\mathbf{O}_{p}(N)\right) \subseteq \mathbf{O}_{p}(N)$. This provides a contradiction, so the proof is finished.

Theorem 5. Suppose that $N$ is a normal solvable subgroup of a group $G$ and suppose that $m$ divides $s$ for every $s \in \operatorname{cs}_{G}(N), s \neq 1$. If $g \in N$ and $\left|g^{G}\right|=m$, then $g \in \mathbf{F}(N)$.

Proof. The hypotheses imply that $\left|\mathbf{C}_{G}(x)\right|$ divides $\left|\mathbf{C}_{G}(g)\right|$ for every $x \in$ $N \backslash \mathbf{Z}(G)$, so by definition we have $g \in M_{p, G}(N)$ for every prime $p \in \pi(N)$. If $|\pi(N)|=1$, then $N$ is nilpotent and the result is trivial, so we can assume $|\pi(N)|>1$. Let us consider the primary decomposition of $g$ in $N$ and we show that if $g_{q}$ denotes the $q$-part of $g$, then $g_{q} \in \mathbf{O}_{q}(N)$ for every prime $q \in \pi(N)$. The claim is trivial when $g_{q} \in \mathbf{Z}(G)$, so we assume that $g_{q} \notin \mathbf{Z}(G)$. Since $\mathbf{C}_{G}(g) \subseteq \mathbf{C}_{G}\left(g_{q}\right)$, then the equality of both centralizers holds by applying the 
hypotheses. Therefore, $g_{q} \in M_{p, G}(N)$ for every prime $p \in \pi(N)$ and by applying Lemma 4, we deduce that

$$
g_{q} \in \bigcap_{p \neq q} \mathbf{O}_{p^{\prime}}(N)=\mathbf{O}_{q}(N) .
$$

As a result, $g \in \mathbf{F}(N)$.

The above theorem might be useful to determine the structure of normal subgroups with three $G$-class sizes, as it happens in [8] when $N=G$. On the other hand, in the proofs of Theorems A and B, we need an application of the following result on coprime action (relying on the Classification of the Finite Simple Groups), in order to obtain information on nonsolvable normal subgroups whose $G$-class sizes are all divisible by some fixed integer.

Theorem 6. Suppose that $A$ and $G$ are finite groups such that $A$ acts coprimely on $G$. If $\mathbf{C}_{G}(A)$ is nilpotent, then $G$ is solvable.

Proof. This appears as Theorem B in [5].

Theorem 7. Suppose that $N$ is a normal nonsolvable subgroup of a group $G$ and suppose that an integer $m$ divides $\left|x^{G}\right|$ for every $x \in N \backslash \mathbf{Z}(N)$. Then $m$ divides $|\mathbf{Z}(N)|$.

Proof. First, we claim that we can assume $\pi(N)=\pi(N / \mathbf{Z}(N))$. If this does not happen, then $N$ can be factorized as a direct product $N=N_{1} \times P$, with $P$ a central Sylow subgroup of $N$ and $N_{1}$ normal in $G$. Then, if $x \in N_{1} \backslash \mathbf{Z}\left(N_{1}\right)$, it trivially follows that $x \in N \backslash \mathbf{Z}(N)$ and since $N_{1}$ is nonsolvable as well, we can apply induction to get that $m$ divides $\left|\mathbf{Z}\left(N_{1}\right)\right|$, which clearly divides $|\mathbf{Z}(N)|$. Thus, the theorem is proved. Now, if $r \in \pi(m)$ such that $r \notin \pi(N)$, we take $R$ a Sylow $r$-subgroup of $G$, which certainly acts coprimely on $N$. From the hypotheses, we deduce that $\mathbf{C}_{N}(R) \subseteq \mathbf{Z}(N)$, and in particular, $\mathbf{C}_{N}(R)$ is nilpotent, so by Theorem 6, $N$ would be solvable, a contradiction. Therefore, $\pi(m) \subseteq \pi(N)$.

The fact that every element of $N \backslash \mathbf{Z}(N)$ has a $G$-class size divisible by $m$ provides the equation

$$
|N|=|\mathbf{Z}(N)|+m k, \quad \text { for some integer } k,
$$

which can be formulated as

$$
|N / \mathbf{Z}(N)|=1+m k /|\mathbf{Z}(N)| .
$$

Since for every $q \in \pi(m)$ we have $q \in \pi(N)=\pi(N / \mathbf{Z}(N))$, then $m_{q}$ divides $|\mathbf{Z}(N)|_{q}$, so $m$ divides $|\mathbf{Z}(N)|$. 
In dealing with normal subgroups which posses exactly two $G$-class sizes, CP-groups appear naturally; these are those groups having all elements of primepower order. The same happens for normal subgroups having exactly two $G$-class sizes of $p$-regular elements, for a prime $p$. We want to remark the usefulness of the study of the $p$-regular class sizes to obtain information on ordinary classes and the structure of a group. We will make use of the following results.

Theorem 8. Suppose that $N$ is a normal subgroup of a group $G$ and that the size of any $G$-conjugacy class contained in $N$ is 1 or $m$, for some integer $m$. Then $N$ is nilpotent.

Proof. This is exactly Theorem 8 of [3].

Theorem 9. If $N$ is a normal subgroup of a group $G$ having two $G$-conjugacy class sizes of p-regular elements, then either $N$ has abelian $p$-complements or all p-regular elements of $N /(N \cap \mathbf{Z}(G))$ have prime-power order.

Proof. This is Theorem 1 of [2].

The structure of finite solvable CP-groups was given by G. Higman fifty years ago, and here we need the structure of nonsolvable CP-groups. This formerly appeared in [4] within the framework of locally finite groups, and was given later by $\mathrm{H}$. Heineken together with the classification of the simple CP-groups.

Theorem 10. If $G$ is a finite, nonsolvable CP-group, then there are normal subgroups $B, C$ of $G$ such that $1 \subseteq B \subseteq C \subseteq G$ and $B$ is a 2-group, $C / B$ is non-abelian and simple, and $G / C$ is a $p$-group for some prime $p$ and cyclic or generalized quaternion.

Proof. This is the main part of Proposition 2 of [10].

Theorem 11. If $G$ is a finite non-abelian simple CP-group, then $G$ is isomorphic to one of the following groups: $\mathrm{L}_{2}(\mathrm{q})$, for $q=5,7,8,9,17, \mathrm{~L}_{3}(4), \mathrm{Sz}(8)$ or $\mathrm{Sz}(32)$.

Proof. This is Proposition 3 of [10].

\section{Proofs of Theorems A and B}

Proof of Theorem B. Suppose that $\operatorname{cs}_{G}(N)=\{1, m, n\}$ with $1<m<n$. We observe that if $m$ does not divide $n$ then Theorem $\mathrm{C}$ of [1] implies that $N$ is solvable. Hence, as $N$ is nonabelian, we have $\mathbf{F}(N / \mathbf{Z}(N))>1$ and the result 
follows. Therefore, for the rest of the proof we will assume that $m$ divides $n$ and we will proceed by induction on the order of $N$.

Let $K / \mathbf{Z}(N)$ be a chief factor of $G$ with $K \leq N$. Notice that $\operatorname{cs}_{G}(K) \subseteq$ $\{1, m, n\}$. Moreover, if $K$ is abelian, then $\mathbf{Z}(N)<K \leq \mathbf{F}(N)$, and the theorem is proved. So, without loss we assume that $K$ is nonabelian. On the other hand, if $K$ is properly contained in $N$, we can apply induction (appealing also to the main result of [3], which establishes the nilpotency of those normal subgroups with exactly two $G$-class sizes) to get $\mathbf{Z}(N) \leq \mathbf{Z}(K)<\mathbf{F}(K) \leq \mathbf{F}(N)$ and the theorem is proved too.

Thus, for the rest we assume $K=N$, that is, $N / \mathbf{Z}(N)$ is a chief factor of $G$ and we will provide a contradiction in this case. We have that $N / \mathbf{Z}(N)$ is a direct product of isomorphic (nonabelian) simple groups, so we write $N / \mathbf{Z}(N)=$ $L_{1} / \mathbf{Z}(N) \times \ldots \times L_{k} / \mathbf{Z}(N)$, where the groups $L_{i} / \mathbf{Z}(N)$ are nonabelian simple and isomorphic. By Remak's Lemma (see for instance $[15,8.9]$ ) it is known that the subgroups $L_{i} / \mathbf{Z}(N)$ are the only minimal normal subgroups of $N / \mathbf{Z}(N)$, and moreover, since this is a chief factor of $G$, we have that $G$ acts transitively by conjugacy on the $L_{i}$ 's. Let $L / \mathbf{Z}(N)$ be one of these simple factors, which in particular is perfect and then $L=L^{\prime} \mathbf{Z}(N)$ and $L^{\prime}=L^{\prime \prime}$. Also, observe that $\mathbf{Z}\left(L^{\prime}\right)=\mathbf{Z}(N) \cap L^{\prime}$. We will make use of the quasisimple group $L^{\prime}$ later.

Now we compute the class size in $\mathbf{N}_{G}\left(L^{\prime}\right)$ of every element in $L^{\prime} \backslash \mathbf{Z}\left(L^{\prime}\right)$. In order to do this we will show first that if $x \in L^{\prime} \backslash \mathbf{Z}\left(L^{\prime}\right)$ then $\mathbf{C}_{G}(x) \subseteq \mathbf{N}_{G}\left(L^{\prime}\right)$. Assume that there exists $g \in \mathbf{C}_{G}(x)$ such that $g \notin \mathbf{N}_{G}\left(L^{\prime}\right)$, then $L^{\prime} \neq L^{\prime g}$ and $L^{\prime} \cap L^{\prime g} \subseteq \mathbf{Z}(N)$. This containment is deduced from the fact that the $L_{i}$ 's are conjugate in $G$, and accordingly, the corresponding derived subgroups are conjugate as well. Hence,

$$
x=x^{g} \in L^{\prime} \cap L^{\prime g} \subseteq \mathbf{Z}(N) \cap L^{\prime}=\mathbf{Z}\left(L^{\prime}\right),
$$

which is a contradiction, as we wanted to prove. Therefore, for every $x \in L^{\prime} \backslash \mathbf{Z}\left(L^{\prime}\right)$ we have

$$
\left|G: \mathbf{C}_{G}(x)\right|=\left|G: \mathbf{N}_{G}\left(L^{\prime}\right)\right|\left|\mathbf{N}_{G}\left(L^{\prime}\right): \mathbf{C}_{\mathbf{N}_{G}\left(L^{\prime}\right)}(x)\right| .
$$

In particular, by applying the hypotheses of the theorem, every element in $L^{\prime} \backslash$ $\mathbf{Z}\left(L^{\prime}\right)$ satisfies that its class size in $\mathbf{N}_{G}\left(L^{\prime}\right)$ is equal to $m / t$ or $n / t$, where $t=\mid G$ : $\mathbf{N}_{G}\left(L^{\prime}\right) \mid$. We distinguish two possibilities: when there are classes of size $m / t$ and when there are not.

Assume first that there exists some element $x \in L^{\prime} \backslash \mathbf{Z}\left(L^{\prime}\right)$ with $\left|x^{G}\right|=m$, which is the same to say that $\left|x^{\mathbf{N}_{G}\left(L^{\prime}\right)}\right|=m / t$. Let us consider the class size of $x \mathbf{Z}\left(L^{\prime}\right)$ in the factor group $L^{\prime} / \mathbf{Z}\left(L^{\prime}\right)$. This class size obviously divides $\left|x^{L^{\prime}}\right|$ and 
also, this divides $\left|x^{\mathbf{N}_{G}\left(L^{\prime}\right)}\right|=m / t$. On the other hand, we know by Theorem 7 that $m / t$ divides $\left|\mathbf{Z}\left(L^{\prime}\right)\right|$, which divides the order of the Schur multiplier of the simple group $L^{\prime} / \mathbf{Z}\left(L^{\prime}\right)$. We conclude then that the simple group $L^{\prime} / \mathbf{Z}\left(L^{\prime}\right)$ has a conjugacy class whose size divides the order of its Schur multiplier. This cannot happen by Lemma 1 and this case is finished.

Finally, assume that all elements in $L^{\prime} \backslash \mathbf{Z}\left(L^{\prime}\right)$ have class size $n / t$ in $\mathbf{N}_{G}\left(L^{\prime}\right)$. By Theorem 7 , we have that $n / t$ divides $\left|\mathbf{Z}\left(L^{\prime}\right)\right|$ and using the same argument in the above paragraph we get a contradiction by appealing again to Lemma 1 .

Proof of Theorem A. We argue by induction on the order of $N$ and divide the proof into the following steps.

Step 1. We can assume that $\mathbf{O}_{q}(N)=\mathbf{Z}(N)_{q}$ for every prime $q \in \pi(m)$. Furthermore, $\mathbf{F}(N)=\mathbf{O}_{p}(N) \times \mathbf{Z}(N)_{p^{\prime}}$, with $\mathbf{O}_{p}(N)>\mathbf{Z}(N)_{p}$.

Suppose that there is a prime $q \in \pi(m)$ such that $\mathbf{O}_{q}(N)>\mathbf{Z}(N)_{q}$. Let $x \notin \mathbf{Z}(G)$ be an $r$-element of $N$ for some prime $r \neq q$ and let $Q$ be a Sylow $q$-subgroup of $\mathbf{C}_{G}(x)$. Notice that $Q \times\langle x\rangle$ acts on $\mathbf{O}_{q}(N)$ and we show that $\mathbf{C}_{\mathbf{O}_{q}(N)}(Q) \subseteq \mathbf{C}_{\mathbf{O}_{q}(N)}(x)$. In fact, if $z \in \mathbf{C}_{\mathbf{O}_{q}(N)}(Q)$ is noncentral in $G$, then $\langle Q, z\rangle \subseteq \mathbf{C}_{G}(z)$. However, the hypotheses imply that $\left|\mathbf{C}_{G}(z)\right|_{q}=\left|\mathbf{C}_{G}(x)\right|_{q}$, so $z \in Q$ and consequently, $z \in Q \cap \mathbf{O}_{q}(N) \subseteq \mathbf{C}_{\mathbf{O}_{q}(N)}(x)$, as we wanted to prove. By applying Thompson's $P \times Q$-Lemma, we obtain $x \in \mathbf{C}_{N}\left(\mathbf{O}_{q}(N)\right)$. Therefore, we have proved that $\left|N: \mathbf{C}_{N}\left(\mathbf{O}_{q}(N)\right)\right|$ is a $q$-power, and on the other hand, notice that $\mathbf{C}_{N}\left(\mathbf{O}_{q}(N)\right)$ is solvable by induction (and Theorem 8), so we conclude that $N$ is solvable too. The first statement of the step is proved.

Now, by Theorem B we can assume that there is a prime $r$ dividing the order of $G$ such that $\mathbf{O}_{r}(N)>\mathbf{Z}(N)_{r}$. However, notice that $r=p$, otherwise, it follows that $r$ does not divide any class size in $N$, and thereby, $N$ has a central Sylow $r$-subgroup, and the theorem follows by induction. Therefore, $\mathbf{F}(N)=$ $\mathbf{O}_{p}(N) \times \mathbf{Z}(N)_{p^{\prime}}$.

Step 2. Every element in $N$ of index $m$ lies in $\mathbf{F}(N)$.

Let $x \in N$ be an element of index $m$. Since $p$ does not divide $m$, then $\mathbf{C}_{G}(x)$ contains some Sylow $p$-subgroup of $G$. In particular, $\mathbf{O}_{p}(N) \subseteq \mathbf{C}_{N}(x)$, which means that $x \in \mathbf{C}_{N}\left(\mathbf{O}_{p}(N)\right)<N$. By induction (and Theorem 8), this centralizer is solvable, and by applying Theorem 5, we get $x \in \mathbf{F}\left(\mathbf{C}_{N}\left(\mathbf{O}_{p}(N)\right)\right) \subseteq \mathbf{F}(N)$.

Step 3. There are no $\pi(m)$-elements in $N$ of index $m$. As a consequence, we can assume that every $p^{\prime}$-element of $\left.N /(\mathbf{Z}(G) \cap N)\right)$ has prime power order. In particular, every $p^{\prime}$-element of $N / \mathbf{Z}(N)$ has prime power order.

Suppose that $w$ is a $\pi(m)$-element in $N$ of index $m$. We can assume that 
$w$ has prime power order by the maximality of its centralizer, say for instance, that $w$ is an $r$-element for some prime $r \in \pi(m)$. By Step 2, we have $w \in \mathbf{F}(N)$, so $w \in \mathbf{Z}(N)$ by Step 1. Now, we observe that any $r^{\prime}$-element of $N$ has index 1 or $p^{a}$ in $\mathbf{C}_{G}(w)$. This is because if $y$ is an $r^{\prime}$-element of $N$, then $\mathbf{C}_{G}(w y)=$ $\mathbf{C}_{G}(w) \cap \mathbf{C}_{G}(y)$ and hence

$$
\left|G: \mathbf{C}_{G}(w y)\right|=\left|G: \mathbf{C}_{G}(w)\right|\left|\mathbf{C}_{G}(w): \mathbf{C}_{G}(w) \cap \mathbf{C}_{G}(y)\right| .
$$

In particular, the index in $N$ of any $r^{\prime}$-element of $N$ (notice that $N \subseteq \mathbf{C}_{G}(w)$ ) is an $r^{\prime}$-number as claimed, and by an elementary result (see for instance [6]), $N$ factorizes as a direct product of an $r$-group $R$ and an $r^{\prime}$-group $H$. Notice that $H \unlhd G$, so by applying induction, $H$ is solvable, whence $N$ is solvable too.

Thus, we have just proved that any $p^{\prime}$-element of $N$ has index 1 or $m p^{a}$ in $G$. By applying Theorem 9, either $N$ has abelian $p$-complements (and thus $N$ is solvable) or every $p^{\prime}$-element of $\left.N /(\mathbf{Z}(G) \cap N)\right)$ has prime power order.

Step 4. $N / \mathbf{F}(N)$ is a CP-group.

Let $z \in N$ such that $\bar{z}=z \mathbf{F}(N)$ has composite order in $N / \mathbf{F}(N)$, so $z \mathbf{Z}(N)$ is also a composite element in $N / \mathbf{Z}(N)$. By Step 3, there exists only one prime $q \neq p$ such that $z \mathbf{Z}(N)$ is a $\{p, q\}$-element. This means that when we consider the primary decomposition of $z$, then the $p$-part and the $q$-part of $z$, say $z_{p}$ and $z_{q}$, are the only factors that do not belong to $\mathbf{Z}(N)$. Since $q$ divides $m$, by Step 3 we have that $z_{q}$ has index $m p^{a}$. Therefore, $\mathbf{C}_{G}(z)=\mathbf{C}_{G}\left(z_{q}\right) \subseteq \mathbf{C}_{G}\left(z_{p}\right)$ and two possibilities arise. Suppose first that $\mathbf{C}_{G}\left(z_{q}\right)=\mathbf{C}_{G}\left(z_{p}\right)$. Then, by Thompson's $P \times Q$-Lemma, we obtain $z_{q} \in T:=\mathbf{C}_{N}\left(\mathbf{O}_{p}(N)\right)$. Now, the normal subgroup $T$ is solvable by induction (and Theorem 8). Notice that $\mathbf{O}_{p}(T) \subseteq \mathbf{O}_{p}(N)$, so in particular $z_{q} \in \mathbf{C}_{N}\left(\mathbf{O}_{p}(T)\right)$. Since $\mathbf{F}(T) \subseteq \mathbf{F}(N)=\mathbf{O}_{p}(N) \times \mathbf{Z}(N)_{p^{\prime}}$, we have $z_{q} \in \mathbf{C}_{T}(\mathbf{F}(T)) \subseteq \mathbf{F}(T)$ because $T$ is solvable, and this implies that $\bar{z}$ is a $p$-element, a contradiction. The other case is when $\mathbf{C}_{G}\left(z_{q}\right)<\mathbf{C}_{G}\left(z_{p}\right)$, which forces that $z_{p}$ has index $m$. By Step $2, z_{p} \in \mathbf{F}(N)$ and thus, $\bar{z}$ has $q$-order, a contradiction too.

Step 5. Conclusion

We work by contradiction and it is assumed that $N$ is nonsolvable. By applying Theorem 10 to $N / \mathbf{F}(N)$ and taking into account that $N$ is a perfect group (otherwise, by induction $N^{\prime}$ would be solvable), just one of the two following possibilities occurs:

i) $\mathbf{O}_{2}(N / \mathbf{F}(N))>1$ and $(N / \mathbf{F}(N)) / \mathbf{O}_{2}(N / \mathbf{F}(N))$ is simple. Let $M / \mathbf{F}(N):=$ $\mathbf{O}_{2}(N / \mathbf{F}(N))$. We can assume that $p \neq 2$, since if $p=2$, then by Step 1 $M=\mathbf{F}(N)$, a contradiction. Thereby, we can take $S$ a Sylow $s$-subgroup of 
$N$, for some prime $s \in \pi(N / \mathbf{F}(N))$ distinct from $p$ and 2 , and we notice that $S$ acts coprimely on $M / \mathbf{Z}(N)$. By coprime action properties, there exists a $S y-$ low 2-subgroup of $M / \mathbf{Z}(N)$, say $T / \mathbf{Z}(N)$, such that $S / \mathbf{Z}(N)_{s}$ acts coprimely on $T / \mathbf{Z}(N)$. Furthermore, as every $p^{\prime}$-element of $N / \mathbf{F}(N)$ has prime power order by Step 3, then the above action is fixed point free. Then, as $s$ is odd, $S / \mathbf{Z}(N)_{s}$ must be cyclic, and so $S$ is abelian. We can apply Taunt's theorem (see for instance [11, 17.7]) in these cases so as to obtain that $s$ does not divide $\left|N^{\prime} \cap \mathbf{Z}(N)\right|=|\mathbf{Z}(N)|$. On the other hand, note that $s$ divides $m$; otherwise, $s$ does not divide any class size of $N$ and consequently, $N$ would have a central Sylow $s$-subgroup, which is not possible. As we know by Theorem 7 that $m$ divides $|\mathbf{Z}(N)|$, this provides a contradiction.

ii) $N / \mathbf{F}(N)$ is a simple CP-group, so it is isomorphic to one of the groups listed in Theorem 11. Note that all of them except just one, $\mathrm{L}_{2}(9) \cong \mathrm{A}_{6}$ (the alternating group of degree 6), have cyclic Sylow $r$-subgroups and $s$-subgroups for two distinct odd primes $r$ and $s$. In particular, they posses cyclic Sylow $r$ subgroups for a prime $r \neq p$, and by applying Taunt's theorem again, we can get a contradiction. Therefore, there is only one simple group to study left: $\mathrm{A}_{6}$. Recall that the Sylow 5-subgroups of $A_{6}$ are cyclic, while its Sylow 2-subgroups and 3-subgroups are not. This implies that we can assume $p=5$, since otherwise, by using the same argument we get a contradiction.

Finally, let us assume that $N / \mathbf{F}(N) \cong \mathrm{A}_{6}$ and $p=5$, and let us consider the factor group $N / \mathbf{O}_{5}(N)$. Note that by Step 1

$$
\mathbf{F}(N) / \mathbf{O}_{5}(N) \subseteq \mathbf{Z}\left(N / \mathbf{O}_{5}(N)\right) \subseteq N / \mathbf{O}_{5}(N)
$$

and

$$
\frac{N / \mathbf{O}_{5}(N)}{\mathbf{F}(N) / \mathbf{O}_{5}(N)} \cong N / \mathbf{F}(N),
$$

which is simple. Then $\mathbf{Z}\left(N / \mathbf{O}_{5}(N)\right)=\mathbf{F}(N) / \mathbf{O}_{5}(N) \cong \mathbf{Z}(N)_{5^{\prime}}$. As $N$ is perfect, we have that $N / \mathbf{O}_{5}(N)$ is a quasisimple group (with associated simple group $\mathrm{A}_{6}$ ). Then the order of its center, that is, $\left|\mathbf{Z}(N)_{5^{\prime}}\right|$ must divide the order of the Schur multiplier of $\mathrm{A}_{6}$, which is 6 . On the other hand, from the fact that every class size of $N / \mathbf{F}(N)$ (which are $\{1,40,45,72,90\}$ ) divides some class size of $N$, and consequently, divides $5^{a} m$, we deduce that 360 divides $5^{a} m$. So, in particular, 72 divides $m$. However, by Theorem 7 we know that $m$ divides $|\mathbf{Z}(N)|$ and this leads to a contradiction. 


\section{References}

[1] Z. Akhlaghi, A. Beltrán, M. J. Felipe and M. Khatami, Structure of normal subgroups with three G-class sizes, Monatsh. Math. 167 (1) (2012), 1-12.

[2] Z. Akhlaghi, A. Beltrán, M. J. Felipe and M. Khatami, Normal subgroups and p-regular G-class sizes, J. Algebra 336 (2011), 236-241.

[3] E. Alemany, A. Beltrán and M. J. Felipe, Nilpotency of normal subgroups having two G-class sizes, Proc. Amer. Math. Soc. 139 (2011), 2663-2669.

[4] R. Brand, Finite groups all of whose elements are of prime power order, Boll. Un. Mat. Ital. $A$ (5) 18, no. 3 (1981), 491-493.

[5] A. Beltrán, Action with nilpotent fixed point subgroup, Arch. Math. (Basel) 69 (1997), 177-184.

[6] A. R. CAmina, Arithmetical conditions on the conjugacy class numbers of a finite group, J. London Math. Soc (2) 5 (1972), 127-132.

[7] A. R. Camina, Finite groups of conjugate rank 2, Nagoya Math. J. 53 (1974), 47-57.

[8] S. Dolfi and E. Jabara, The structure of finite groups of conjugate rank 2, Bull. Lond. Math. Soc. 41 (2009), 916-926.

[9] R. Gorenstein, R. Lyons and R. Solomon, The Classification of the Finite Simple Groups, Vol. 40, Number 3, Mathematical Surveys and Monographs, American Mathematical Society, 1998.

[10] H. Heineken, On groups all of whose elements have prime power order, Math. Prod. R. Ir. Acad. 106A (2) (2006), 191-198.

[11] B. Huppert, Character Theory of Finite Groups, Vol. 25, De Gruyter Expositions in Mathematics, Berlin, New York, 1998.

[12] I. M. IsAacs, Finite Group Theory, Vol. 92, Graduate Studies in Mathematics, American Mathematical Society, 2008.

[13] N. Itô, On finite groups with given conjugate types, II, Osaka J. Math. 7 (1970), 231-251.

[14] P. Kleidman and M. Liebeck, The Subgroup Structure of the Finite Classical Groups, Vol. 129, London Mathematical Society Lecture Note Series, Cambridge University Press, Cambridge, 1990.

[15] J. H Rose, A course on Group Theory, Cambridge University Press, Cambridge, 1978.

[16] J. Rebmann, F-Gruppen, Arch. Math. 22 (1971), 225-230 (in German).

ANTONIO BELTRÁN

DEPARTAMENTO DE MATEMÁTICAS

UNIVERSIDAD JAUME I

12071 CASTELLÓN

SPAIN

E-mail: abeltran@mat.uji.es

MARÍA JOSÉ FELIPE

INSTITUTO UNIVERSITARIO

DE MATEMÁTICA PURA Y APLICADA

UNIVERSIDAD POLITÉCNICA DE VALENCIA

46022, VALENCIA

SPAIN

E-mail: mfelipe@mat.upv.es

(Received May 15, 2012; revised November 27, 2012) 